\title{
APRENDIZAJE BASADO EN PROYECTOS (ABP) ANTE EL RETO DE UNA NUEVA ENSEÑANZA DE LAS CIENCIAS
}

José Manuel Bautista-Vallejo bautista@uhu.es

0000-0002-4238-9507

Universidade de Huelva, Espanha

Manuel Jesús Espigares-Pinazo manueljesus.espigares@unir.net manueljesus.espigares@unir.net Universidad Internaciona

Rioja España.

Rafael Manuel Hernández-Carrera rmhercar@upo.es

0000-0002-7722-8105

Universidad Pablo de Olavide, Sevilla España.
RESUMO

Este trabajo presenta una revisión del modelo de Aprendizaje Basado en Proyectos (ABP) y su relación con la nueva enseñanza de las ciencias. Los proyectos de trabajo tienen especial interés en desarrollar un proceso de enseñanza-aprendizaje basado en la colaboración, la comunicación, la investigación, el pensamiento crítico y la resolución de problemas. Por su parte, dentro de la ciencia la experimentación, la medición de fenómenos, la manipulación de variables, etc., son acciones que permiten un acercamiento racional a los fenómenos de la naturaleza. En este trabajo se reflexiona por qué, entonces, el modelo ABP permite un marco interesante para el desarrollo de estas cualidades científicas en las escuelas.

PALAVRAS-CHAVE: Aprendizaje Basado en Proyectos, ciencia, didáctica, colaboración, pensamiento crítico. 


\section{Introducción}

La pedagogía del proyecto y la optimización del clima educativo de la clase son dos dominios de investigación que han vuelto a ser retomados para advertir y resolver muchos de los problemas de la enseñanza de hoy.

En el caso de las ciencias, la metodología Aprendizaje Basado en Proyectos ( $A B P$, en adelante; Project-Based Learning, PBL, en inglés) parece prometedora por cuanto que permite convertir en proyectos todos los contenidos científicos los cuales se diseñan y resuelven de forma creativa y, consecuentemente, permite que los alumnos sean capaces de aplicar los conocimientos en el mundo real, usando lo que han aprendido para resolver problemas, responder a preguntas complejas y crear recursos y productos innovadores y de calidad.

Las "competencias para el éxito" en las que deberían centrarse las propuestas didácticas basadas en ABP son: resolución de problemas y preguntas complejas, creación de hábitos intelectuales y desarrollo de cualidades personales (perseverancia, creatividad o trabajo autónomo y en equipo).

No debemos llevarnos a error al hablar de pedagogía del proyecto, pues una observación sobre el terreno de las prácticas que llevan esta etiqueta pedagógica, permiten constatar de una manera rápida que el $A B P$ se trata tanto de una pedagogía del "tema" como de una pedagogía del descubrimiento guiado.

Una pedagogía del proyecto es una manera genérica de entender el trabajo educativo de proyectos. Como tal designa variadas formas no necesariamente independientes: proyectos de trabajo, proyectos de formación, proyectos cooperativos, proyectos educativos. En todos, la transacción (es decir, el conjunto de los modos después de la emergencia del deseo de hacer cualquier cosa hasta el establecimiento del contrato), la negociación, el contrato (o mejor, la sucesión de contratos), los acuerdos, el trabajo autónomo y en equipo, la elaboración y resolución de preguntas complejas, etc., son conceptos muy importantes en la relación pedagógica y en la pedagogía del proyecto, estando en el origen de su definición.

Esto indica precisamente que no cualquier cosa es un proyecto. Por ejemplo, un proyecto educativo censurado por un director e impuesto al personal de una organización, o un proyecto de trabajo colectivo concebido por un enseñante e impuesto a sus alumnos, etc., en ningún caso deberían ser considerados bajo el nombre de "Pedagogía del Proyecto".

Los proyectos de trabajo, individuales o colectivos, donde las características finales y las modalidades de producción son enunciadas en un contrato o sucesión de contratos, saltan cualitativamente sobre otros métodos de trabajo en las organizaciones educativas, en el sentido de que el alumnado aprende a ser consciente acerca de su aprendizaje, que no es decir poco. Aunque ello no puede ser medido en sentido estricto, sí que puede ser tentativamente formulado de manera que tanto el profesorado como el alumnado puedan comprender lo que hacen.

Como afirma Dzib Goodin (2013), el tema del aprendizaje ha sido ampliamente estudiado desde distintas perspectivas y en años recientes. El estudio de nuestras redes neuronales nos ha permitido conocer cómo la naturaleza ha dotado a los sistemas con la habilidad para adaptarse al medio, que es la línea 
que permite la pervivencia de las especies, lo cual puede ser clave para la comprensión del aprendizaje y su necesidad de desarrollarse como un sistema flexible.

Esta visión del aprendizaje demanda nuevas formas de trabajo en los espacios educativos, ante la certeza de que medios ricos y complejos, pero convenientemente planificados, pueden provocar formas extremadamente ricas de desarrollo de nuestro cerebro y, consiguientemente, capacidad de aprendizaje más satisfactorio.

La pedagogía del proyecto se relaciona con todos aquellos métodos que entienden la mediación de los recursos, tanto personales como materiales, docentes, objetos, medios, métodos, espacios, tiempos, etc., como contrarios al sentido del alumnado como un receptáculo vacío que precisa ser llenado con el conocimientos que se concretan en el currículum explícito que se desarrolla y que emanan del profesor, en donde la instrucción hace hincapié en la dependencia del alumnado y la educación es considerada como creadora de un individuo mucho más autónomo (MORALES y TORRES, 2015).

Al contrario que esto, la pedagogía del proyecto está más interesada en facilitar los procesos del aprendizaje, en dejar una huella en la capacidad de enganchar al alumnado en torno a un tema que le motiva y que satisface su interés por explorar nuevos conocimientos. Esto parece decir poco, pero es decir mucho.

\section{Pedagogía del proyecto en el aula}

Tanto en el ámbito nacional como en el internacional encontramos hoy una gran variedad de modelos de trabajo y de enseñanza en las escuelas. La pedagogía parece haberse desarrollado hasta niveles antes desconocidos. Si bien esto, una de las más frecuentes críticas que puede hacerse al sistema educativo es su falta de innovación, su incapacidad para desarrollar estrategias que despierten la capacidad de ser creativos y resolver problemas (CRAFT ET AL., 2014). Ambos polos son representativos de las instituciones educativas de hoy.

La pedagogía del proyecto forma parte de los llamados modelos de trabajo y enseñanza (teaching and working patterns), los cuales son entendidos como la combinación única de actividades del profesor, actividades del alumno y la distribución de los procesos de decisión y control del proceso global en una unidad de aprendizaje (ARENDS, 2014). En el sentido más simple de lo que significan los modelos de trabajo y enseñanza, en la riqueza que representa la variedad de los mismos, son muy conocidos y extendidos a nivel internacional como métodos de resolución de problemas individuales y de la enseñanza.

En concreto, los proyectos de trabajo, como método, dentro de su variedad aplicativa, se enmarcan en aquellas acciones de enseñanza-aprendizaje de corte innovador e investigativo (GARRIGÓS SABATÉ y VALERO GARCÍA, 2012).

Los proyectos de trabajo han llegado a ser parte del trabajo de la escuela desde finales de los años setenta. Sin embargo, éstos han sido menos extendidos que otros modelos de trabajo, como por ejemplo los tópicos de trabajo (topic works) y los talleres de enseñanza (working-teaching). La razón la encontramos en que desde los años setenta este modelo vino de la mano de la educación de adultos (BROOKFIELD, 2013), lo que hizo que en su desarrollo llegara a adquirir 
características propias de la educación de adultos, que lo hiciera de más difícil aplicación en el contexto de la Educación Primaria y Secundaria.

Si bien existe una gran cantidad de modelos educativos, los cuales tienen sus ventajas e inconvenientes en su aplicación didáctica y educativa, el mero hecho de la existencia de esa variedad no determina el éxito en la enseñanza. Incluso la pedagogía del proyecto no es la respuesta a todos los problemas de la enseñanza y el aprendizaje, lo que no quita el extraordinario poder que esta metodología tiene para la generación de ideas, el desarrollo de la creatividad, el trabajo en equipo, la resolución de problemas y la adquisición del conocimiento trabajando sobre los contenidos de forma activa (MORALES y TORRES, 2015).

\section{Conceptualización y principios del ABP}

Se han ensayado muchas definiciones de ABP, una de ellas podría ser ésta: se trata de un modelo de acción y enseñanza-aprendizaje en el cual los alumnos activamente y con un propósito personal muy claro investigan su entorno, forman ideas y conceptos acerca de éste, tratan de comprenderlo, forman opiniones acerca de él y actúan en él.

En términos de contenido, determinadas áreas problemáticas son seleccionadas y examinadas desde diferentes puntos de vista y, de esta manera, son tratadas ambas histórica y contemporáneamente desde una visión que ayuda a los alumnos a adquirir una capacidad de discernimiento para la vida diaria, al tiempo que pueden hacer de su vida en sociedad algo claro y comprensible.

En un análisis de las distintas prácticas de $A B P$ se comprueba que en esta metodología los alumnos llevan a cabo un amplio proceso de investigación para responder a una pregunta compleja, a un problema o a un cambio (BENDER, 2012). Igualmente, los alumnos tienen autonomía y capacidad de decisión en el desarrollo de los proyectos. Al mismo tiempo, estos tienen que ser planeados, diseñados y elaborados para conseguir que los alumnos aprendan los contenidos básicos, trabajen importantes competencias como por ejemplo la colaboración, la comunicación, el pensamiento crítico y la resolución de problemas y creen productos y presentaciones de calidad, donde demuestren su capacidad de comunicación, etc. (HEYDRICH, ROJAS y HERNÁNDEZ, 2012; MORALES y TORRES, 2015).

El núcleo central del ABP es el alumnado y su actividad. Para que esta actividad sea promovida debe haber proyectos que la guíen y permitan su desarrollo. En su nivel más general, un proyecto de trabajo puede estar gobernado por los siguientes principios (HEYDRICH, ROJAS y HERNÁNDEZ, 2012; STEFANOU ET AL., 2013; MORALES y TORRES, 2015; TRAVERSO-RIBÓN ET AL., 2016): 1) Orientación hacia el problema; 2) Orientación hacia el producto; 3) Regla colaborativa (dirección y cooperación conjunta entre profesor y alumno); 4) Interdisciplinariedad; 5) Acerca de la selección del contenido ejemplar. 
La consideración del problema o asunto a resolver es importante por cuanto que el alumnado debe desear poner luz o desear resolver el problema en cuestión. Instaurar este sentimiento en el alumnado es vital para todo proyecto por cuanto que es el punto de partida para desarrollar un proceso de aprendizaje basado en un proyecto donde el alumno desea poder manejar el conocimiento con soltura (PREETI, ASHISH y SHRIRAM, 2013), cosa que en el momento inicial no acontece.

Por otro lado, el vocabulario que empleamos en nuestra comunicación en este momento inicial es muy importante, siendo las palabras "curiosidad", "duda", "interrogantes" o "preguntas" las más usuales para desarrollar actitudes propicias para el trabajo posterior (HARADA, KIRIO y YAMAMOTO, 2008). La persona que guía el proceso debe, en todo momento, hacer posible que los alumnos adquieran y manejen este vocabulario desde el principio.

Es importante, en este sentido, plantearse algunas cuestiones, las cuales resultan decisivas para el inicio del proyecto:

a) ¿Cómo podemos llevar a los alumnos, en la primera parte del proceso, a situaciones donde lleguen a mostrar curiosidad sobre una cuestión, a preguntarse sobre algo o llegar a ser conscientes de que hay un problema que es susceptible de ser resuelto por ellos?

b) ¿Cómo puede construirse un proceso de trabajo que esté basado en la curiosidad existente en los alumnos y en el sentido de búsqueda de solución a un problema en el que éstos están interesados?

\section{2) Orientación hacia el producto}

Una vez que el alumno ha aprendido algo, trabajado sobre algo o comprendido algo, éste debe expresar lo que ha aprendido y comprendido y lo que piensa acerca de ello, de manera que pueda compartir con otros una determinada adquisición personal o grupal (BILGIN, KARAKUYU y AY, 2015). Si el proceso es importante en el $A B P$, de igual forma el producto final en términos de aprendizaje debe ocupar un lugar estratégico, aprendizaje como adquisición que se vehicula a partir de distintas formas de comunicación. Se trata de un producto que entra en diálogo con otros.

El producto es el medio a través del cual uno presenta algo de cara a comunicar a otros una determinada idea, aplicación o conocimiento más o menos concreto. Esto se realiza a través de palabras, dibujos, mapas mentales, producción de materiales, juegos, etc. Se trata de distintas maneras de expresar, presentar y representar la realidad conocida por los alumnos, donde la idea más potente a manifestarse es la capacidad de comunicación (NOTARI, BAUMGARTNER y HERZOG, 2014). La comunicación con estilo y soporte personal es el hecho primordial que se pone de manifiesto.

3) Regla colaborativa (dirección y cooperación conjunta entre profesor y alumno) 
En el ABP el agente principal de aprendizaje es el alumno, centro de todos los esfuerzos de la institución educativa. Si bien esto, el rol de quienes dirigen, facilitan o median en los proyectos es fundamental. La tarea de los docentes se relaciona con la dirección participativa de los proyectos del alumnado de forma que estos últimos entiendan el camino y tengan una guía para el desarrollo de los proyectos, en un marco de colaboración mutua (LEE ET AL., 2014).

Desde el punto de vista de la dirección participativa, la voz del alumno es muy importante, al igual que la del profesor, unos como otros deben trabajar juntos en lo que respecta tanto a los contenidos como a los procesos.

En esta relación educativa que se establece entre quienes guían y quienes son guiados (SILVA y NAVARRO, 2012), algunas cuestiones fundamentales son: crear una atmósfera de diálogo mutuo y participativo, adoptar y fomentar marcos de decisión para las ideas de los alumnos en cualquiera de las situaciones y maneras de expresión, etc. La conveniencia de este ecosistema de participación libre, espontánea y creativa es un requisito esencial para los proyectos de trabajo y su desenvolvimiento.

4) Interdisciplinariedad

En el intento de comprender un problema o un fenómeno, las relaciones que podamos establecer entre conceptos, puntos de vista, materiales, etc., de diferentes materias es fundamental. Este tipo de relaciones, presentes en la naturaleza de una forma espontánea, deben ser llevadas a esos espacios artificialmente creados que hemos denominado aulas y centros escolares (GOUVEA ET AL., 2013), de forma que cuando se den los procesos de aprendizaje, la relación entre conceptos e ideas pueda circular sin mayor freno.

Realmente la metodología basada en ABP considera la interdisciplinariedad un requisito y, también, un resultado de los proyectos (LYALL y FLETCHER, 2013; PENNINGTON ET AL., 2013). En el primero de los casos, estrategias que posibiliten la interdisciplinariedad presentan mejores estímulos para la creación y la resolución de los problemas propuestos en el proyecto de trabajo. Por otro lado, la propia interdisciplinariedad es un resultado del proyecto por cuanto que el trabajar en un determinado marco de aprendizaje abierto y conectivo establece las condiciones de nuevos y mejores aprendizajes, en entornos competenciales colaborativos muy requeridos hoy (SAAVEDRA y OPFER, 2012).

\section{5) Selección del contenido ejemplar}

El llamado contenido ejemplar es aquel que se presenta en unidades de contenido significativas para el alumnado. Se basa en la necesidad de comprender que la formación siempre se da a largo plazo y que, siendo lo más importante la capacidad de aprendizaje del alumnado, lo cual depende de múltiples factores internos y externos al mismo, la selección de los contenidos es un aspecto relevante en relación a los objetivos educativos planteados (BENDER, 2012).

Los contenidos no se presentan nunca en el proceso formativo de forma aislada, éstos deben estar relacionados de forma coherente y sistémica con unos objetivos y se enmarcan en unidades de espacio y tiempo. Los contenidos tienen 
más sentido cuando todos estos elementos son presentados de forma cuidada, cuando están bien planteados, son significativos y coherentes y responden a criterios lógicos y psicológicos de cara al alumnado. La visión de J. Bruner (2003) de que todos los alumnos son capaces de aprender cualquier cosa siempre y cuando se haga en su nivel de representación es un fundamento de este principio.

Es necesario, por tanto, dar respuesta a las preguntas sobre la elección del tema, de los contenidos del proyecto, qué importancia tiene, etc. Igualmente la pregunta del porqué es fundamental (SINEK, 2009). Este autor considera que nuestro comportamiento se ve afectado por las conjeturas o por las verdades percibidas, es decir, tomamos decisiones basándonos en lo que pensamos que sabemos. En este sentido, empezar todo proyecto por un porqué nos ayuda a entender por qué hacemos lo que hacemos, cuál es el propósito, la causa, las creencias que tenemos en relación a nuestra actividad proyectiva con el alumnado.

\section{El modelo "IVAC" (Investigación, Visiones, Acciones y Cambios)}

Se han descrito múltiples posibilidades de proyectos de trabajo, la mayoría de los cuales siguen unos principios como los descritos, si bien igualmente se presentan con diferencias notorias cada uno de ellos (PREETI, ASHISH y SHRIRAM, 2013; LEE ET AL., 2014).

En el contexto aplicativo del enfoque ABP, el modelo IVAC (Investigación, Visiones, Acciones y Cambios), empleado por Jensen (1997) para estructurar y facilitar la participación del alumnado, parte de la idea de que el alumnado está totalmente involucrado en la elección de los temas que van a trabajar, a un tiempo que investigan activamente y responden sobre el sentido y la importancia que ese tópico, problema o tema tiene para ellos.

De igual forma, la manera en que los alumnos introducen determinadas visiones del problema en cuestión que se está investigando y el contenido mismo de esas visiones, contribuye a promover una cultura en torno a las diferentes visiones y al necesario diálogo que debe producirse sobre la base de esas diferentes alternativas interpretativas: qué opinión tienen los alumnos, cuál es la solución que proponen, qué mecanismos sugieren para el futuro, etc.

El contexto propicio de esta forma de expresar un proyecto de trabajo deja vía libre para discutir las propuestas de los alumnos sobre las visiones aportadas, sobre los posibles cambios a acometer, para así descubrir lagunas de conocimiento, las barreras que entorpecen la posibilidad de cambio, la complejidad y riqueza de la realidad, etc.

En este modelo, el profesorado y otros miembros de la plantilla involucrados en el proyecto tienen el rol de responsables facilitadores de las actividades del proyecto, con las tareas de inspirar, apoyar y presentar continuos desafíos al alumnado (JENSEN, 1997; NORDIN, 2016).

\section{Las fases del modelo IVAC y la pedagogía de la pregunta}

Es necesario poner de relieve la importancia de la pedagogía de la pregunta para el desarrollo de un pensamiento profundo, auto dirigido, reflexivo y sistemático. El uso de la misma permite el logro de un cambio de una conciencia 
pasiva a una conciencia crítica (OCHOA, 2013; CAMARGO y USECHE FIGUEREDO, 2015), aspecto siempre relevante en el desarrollo de los proyectos. Esta es la razón por la que un proyecto debe estar inmerso en marcos de trabajo en donde las preguntas sean habituales, las reflexiones permanentes y los interrogantes que promuevan intentos de respuesta constantes (OCHOA, 2013).

Jensen (1997) y Jensen y Simovska (2005) contemplan este modelo de ABP con aportaciones de diversos grupos y perspectivas de cara al trabajo colaborativo con jóvenes. Los aportes del mismo son los siguientes:

a) Investigación de un tema

La investigación, en el sentido amplio del término, es un elemento fundamental de todo proyecto. Un proyecto debe invitar a investigar permanentemente, en cada una de sus fases de desarrollo, en una demostración permanente de que la investigación es una competencia (MORALES y TORRES, 2015) y, además, una actitud con la que afrontamos cada uno de los pasos que vamos dando en el proyecto. Algunas preguntas que pueden guiar e inspirar esta capacidad en el proyecto pueden ser: ¿Por qué es importante para nosotros? ¿Es importante para nosotros o para otros, ahora o para el futuro? ¿Influyen el estilo y las condiciones de vida? ¿Qué influencias estamos teniendo en cuenta? ¿Cómo fue esto en el pasado y por qué cambió?

\section{b) Desarrollo de las visiones}

Un proyecto de trabajo tienen sentido porque aunque las decisiones a lo largo del proyecto se van tomando colaborativamente, es lo cierto que se pone en la capacidad de decidir del alumno una buena parte de las posibilidades en la toma de decisiones (RAVITZ ET AL., 2012).

Esto provoca dos actitudes: por un lado, el aumento de la seguridad en el alumnado, que ve crecer su capacidad de toma de decisiones y comprueba que sus ideas y palabras son tenidas en cuenta; por otro lado, la posibilidad de que las distintas visiones entren en relación, de forma que en ese marco dialógico las relaciones entre conceptos e ideas posibilitan un entorno más creativo para la resolución de los problemas.

Algunas preguntas que pueden guiar e inspirar esta capacidad en el proyecto pueden ser: ¿Qué alternativas podemos imaginar? ¿Cuáles son las condiciones en otros países y en otras culturas? ¿Qué preferimos y por qué?

e) Acción y cambio

Este tercer grupo se ocupa de elegir y justificar los planes de acción que deben adoptarse para provocar cambios positivos en relación con el problema de que se trate (JOHNSON y DELAWSKY, 2013), incluido el análisis de las posibles barreras y estrategias para superarlos.

Algunas preguntas que pueden guiar e inspirar esta capacidad en el proyecto pueden ser: ¿Qué cambios traeremos más relacionados con estas visiones? ¿Cambios en nuestra vida, en la clase, en la sociedad? ¿Qué posibilidades de acción existen para alcanzar estos cambios? ¿Cuáles son las barreras a suprimir en relación a estos cambios? ¿Cuáles son las barreras entre acción y cambio? ¿Qué acciones suprimiremos? ¿Cómo queremos evaluar estas acciones? 


\section{Producción de conocimiento y enseñanza de las ciencias}

Las apreciaciones sobre el tipo de sociedad en la que estamos son constantes. Sobre si debe ser definida como la sociedad de la inteligencia, del aprendizaje, del conocimiento, de la tecnología, etc., simplemente los distintos autores no se ponen de acuerdo (FAGERBERG, LANDSTRÖM y MARTIN, 2012). En este momento el mundo es campo abonado de profundos cambios de naturaleza social, económica, epistémica, tecnológica y cultural, de los cuales pueden extraerse, al menos, dos consideraciones generales:

Primero, la consideración de la pregunta sobre el tipo de aprendizaje para un futuro desconocido. Por un lado, nuestros propios sistemas de conocimiento y tecnologías están produciendo un cambio reflexivo, en el cual lo producido se presenta como catalizador de futuros cambios. Es así que este elemento reflexivo está en el nexo de unión entre el conocimiento y el cambio que se produce siempre más rápido, provocando consiguientemente una situación de cambio esencialmente desconocible (BARNETT, 2004). Entonces, ¿cuál es la idea que tenemos de aprendizaje?

En este sentido, Alma Dizb (2013) entiende que el proceso de aprendizaje no depende de los educadores, es una función otorgada a todas las especies sobre la faz de la tierra con fines de pervivencia, con el objetivo de responder lo más adaptativamente posible ante el entorno. Es decir, el aprendizaje es un proceso personal que puede ser limitado o engrandecido por el entorno.

Segundo, que la reflexividad construida de nuestros sistemas de conocimiento tiene repercusiones a nivel individual. Los crecientes niveles de satisfacción en conceptos, ideas y, por supuesto, discursos manejados, necesitan de individuos si ellos de cualquier forma van a dar sentido al mundo que están encarando. Es por ello que la reflexividad a nivel de cada individuo, es decir, la capacidad crítica de irse interrogando sobre el universo que nos rodea, es una necesidad en orden a asimilar y acomodar el nuevo orden.

Esta es la razón por la que el aprendizaje para un mundo desconocido llama, dicho con pocas palabras, a un cambio ontológico y, desde luego, una nueva forma de proceder en la práctica (BARNETT, 2004).

En el ámbito de las ciencias, en un momento en que se perciben cambios en la estructura de los saberes, cambios en las formas tradicionales de organización, división y especialización del conocimiento, transformación de la circulación y apropiación del conocimiento y cambios en el papel social de las profesiones, es necesario abordar estos cambios con un nuevo marco de trabajo en donde estas peculiaridades sean tenidas en cuenta.

En el caso de las universidades ya advertía Barnett (2001) que al integrarse más con los criterios cognitivos de la sociedad, ésta se ve obligada a mostrar nuevas definiciones sobre lo que se debe conocer y aprender. ¿Cuál es, entonces, el aporte de un modelo basado en ABP en el marco de esta nueva situación? 
Como se ha mencionado, la base de este método de trabajo es la continua discusión crítica, el debate y el diálogo entre agentes, es decir, entre profesoralumno y alumnos-alumnos (BENDER, 2012). Esta metodología será la herramienta de la que los implicados en el proceso enseñanza-aprendizaje se sirven para la construcción continua de sus conocimientos. De esta forma se consigue el desarrollo y la evolución del pensamiento de los alumnos a través del uso de la capacidad crítica y reflexiva individual.

Se añade a la discusión y diálogo permanentes la referencia al grupo, equipo de trabajo, la pluralidad y variedad de pensamientos y visiones. En efecto, una de las principales características de este modelo de enseñanza-aprendizaje es la referencia manifiesta, en todo instante, al trabajo en grupo y los beneficios que esta peculiaridad conlleva. Estudios sobre los logros en el ámbito de las ciencias, tecnología, ingeniería y matemáticas (STEM por sus iniciales en inglés), así lo ponen de manifiesto (MUJIKA ET AL., 2014; HAN, CAPRARO y CAPRARO, 2015).

\section{Desarrollo de cualidades facilitadoras}

En lo que respecta al ámbito de los paradigmas y la consideración de la ciencia, temas de especial interés no sólo para los creadores de ciencia sino, también, para aquellos que la difunden y la enseñan, tenemos aún hoy las visiones contradictorias y hasta las luchas encarnizadas que hace más de treinta años se produjeron entre Feyerabend y Gellner (FEYERABEND, 1979; GELLNER, 1979), las cuales se iniciaron tras el optimismo pedagógico de los sesenta, años después de la Segunda Guerra Mundial.

Por su parte, Feyerabend ha sostenido insistentemente que la ciencia no es la empresa racional que cree ser y que su creencia en su propia supremacía y su lugar dominante en la sociedad moderna son formas de ideología. Por su lado, tenemos la postura de Gellner, quien sostiene que la alta apreciación que se otorgó a la ciencia es totalmente adecuada: no sólo social sino también epistemológicamente está más claro el estatus superior de la ciencia en nuestra cultura. Finalmente, Feyerabend sostiene que el estatus superior de la ciencia carece de fundamento epistemológico y representa el dominio de un estrecho agrupamiento cognitivo, que cierra de manera inapropiada nuestras opciones cognitivas.

En este marco, que prosiguió a lo largo de los años, si bien el auge del desarrollo tecnológico ha permitido la idea de supremacía de la tecnología, los métodos han sido estudiados como decisivos para resolver las dudas epistemológicas que pudiera haber en torno a esta discusión (OCHOA, 2013). Indudablemente, toda metodología o método de trabajo utilizado por un profesional dentro de un sistema educativo determinado, con independencia del país al que nos refiramos, tratará de conseguir los objetivos esenciales que el sistema educativo propone, que a grosso modo estarán relacionados con la socialización del individuo y una serie de logros relacionados con su capacidad de aprender (SAAVEDRA y OPFER, 2012). No obstante, y al margen del objetivo u objetivos esenciales mencionados, todo método, intrínsecamente, transmite otras cualidades de las que los individuos y toda la sociedad se beneficiarán. 
Algunas de las cualidades que facilitan los proyectos de trabajo las podemos enumerar en las siguientes (MIHARDI, HARAHAP y SANI, 2013; NOTARI, BAUMGARTNER y HERZOG, 2014; BILGIN, KARAKUYU y AY, 2015):

- Habituar al individuo a trabajar en grupo, a cooperar con compañeros, aceptar y respetar la opinión de los otros.

- Crear discusión pedagógica, con la que el grupo creará conocimiento.

- Usar la capacidad de reflexión individual y grupal.

- Fomentar el debate y la confrontación de diferentes puntos de vista.

- Auto-construcción del conocimiento de cada individuo.

- Desarrollo, fomento y evolución del pensamiento crítico y del criticismo.

- Fomento de la utilización del diálogo interpersonal.

- Desarrollo y fomento de la inteligencia abstracta.

- Otorgar valor de las ideas de los alumnos.

\section{Puesta en práctica}

Cuando un proyecto va a ser organizado, a menudo es necesario realizar una estructuración previa de sus partes: ¿qué vamos a hacer?, ¿cómo lo vamos hacer?, ¿de qué va a constar?, ¿qué queremos conseguir? Un proyecto de trabajo puede ser de pequeña extensión o de larga extensión, contar con más o menos fases, todo ello dependerá de los objetivos que establezcamos y de las peculiaridades que pretendamos conocer, conseguir y desarrollar (BENDER, 2012).

Es de notable importancia que los profesores y los alumnos implicados en este modelo, estén totalmente familiarizados con el contenido y con el proceso metodológico que se pretende seguir para ir alcanzando las diferentes etapas o fases establecidas. Este hecho posibilita una sencilla y buena elaboración del proyecto. No solamente es importante estar familiarizados con el modelo de trabajo, para Capraro y Slough (2008) y Han, Capraro y Capraro (2015) en el caso de los docentes éstos deberían conocer cuáles son las mejores circunstancias de aplicación del mismo.

El uso de recursos bibliográficos y otras fuentes es importante igualmente, pero es un rasgo muy significativo de los proyectos de trabajo que el proceso sea guiado a través de la autorreflexión, de la lectura y del trabajo personal, consiguiendo de este modo absorber, interiorizar y analizar información de manera idiosincrática (JOHNSON y DELAWSKY, 2013), permitiendo en este sentido partir, bajo la luz del debate y discusión pedagógica de los agentes, desde la información primaria hacia la construcción de la propia realidad personal. En este sentido, la auto-regulación y la autonomía se presentan como aspectos importantes del ecosistema en el que trabajar los distintos proyectos (STEFANOU ET AL., 2013).

La pregunta es, entonces, ¿cómo logramos mayor número de individuos interesados por la ciencia? Considerada esta cuestión como elemento previo, pues sin interés no hay dedicación, la manera en que trabajemos, las estrategias usadas, la forma de plantear las hipótesis, la actitud frente al ensayo-error, la propia 
consideración del error, etc., se convierten en los elementos más destacados del ecosistema, de forma que envolviendo todo el trabajo pueda provocarse un verdadero $A B P$.

De otro lado, es necesaria la consideración de la capacidad de investigación de docentes y alumnado en los centros escolares. En el caso del profesorado, sus prácticas profesionales deben ir más allá de la consideración de tarea técnica, para ser calificada como práctica intelectual y autónoma, sólo desde este convencimiento los docentes van a ser capaces de implementar verdaderos proyectos en donde la ciencia genere la capacidad de describir, analizar, criticar e intervenir en la realidad. Competencias como la capacidad de emprender o la capacidad de generar ideas van a depender de estos roles docentes y este ecosistema generado (MORALES y TORRES, 2015).

Finalmente, en el ámbito de las ciencias, la experimentación, la medición de fenómenos y la manipulación de variables, etc., son acciones que permiten un acercamiento racional a los fenómenos de la naturaleza. Estas habilidades, unidas a otras competencias que son importantes para los profesionales del siglo XXI como la colaboración, la comunicación y el pensamiento crítico, son susceptibles de ser trabajadas por un modelo de ABP. La aplicación del ABP posibilita cada una de estas acciones a lo largo de los proyectos, de forma que se van adquiriendo de forma práctica en un marco de reflexión compartida, dialógica y crítica (RAVITZ ET AL., 2012). 


\title{
Project-Based Learning (PBL) facing the challenge of a new science teaching
}

\begin{abstract}
This paper presents a review Project-Based Learning (PBL) model and its relationship with the new science teaching. The project-works are particularly interested in developing a teaching-learning process based on collaboration, communication, research, critical thinking and problem solving. Meanwhile, within science the experiment, measurement of phenomena, manipulation of variables, etc., are actions that allow a rational approach to the phenomena of nature. In this paper we examine why the PBL allows an interesting frame for the development of these scientific qualities in the school.
\end{abstract}

KEYWORDS: Project-Based Learning, science, didactics, collaboration, critical thinking. 
ARENDS, Richard. Learning to teach. Nueva York: McGraW-Hill higher Education, 2014.

BARNETT, RONALD. LOS LíMITES DE LA COMPETENCIA. El CONOCIMIENTO, LA EDUCACIÓN SUPERIOR Y LA SOCIEDAD. BARCELONA: GEDISA, 2001.

BARNETT, RONALD. LEARNING FOR AN UNKNOWN FUtURE. HIGHER EdUCATION RESEARCH \& DeVELoPMENT, V. 23, N. 3, P. 247-260, 2004.

BENDER, WILLIAM N. PROJECT-BASED LEARNING: DIFFERENTIATING INSTRUCTION FOR THE 21ST CEnTURY. Thousand OAKS, CALIFORNIA: CORWIn Press, 2012.

Bilgin, Ibrahim, kARAkUyu, Yunus y ay, Yusuf. The Effects of Project Based Learning on Undergraduate Students' Achievement and Self-EfFicacy Beliefs Towards SCience Teaching. Eurasia Journal of Mathematics, Science \& TeChnology EDUCATION, v. 11, N. 3, P. 469-477, 2015.

BROOKFIELD, Stephen D. PoWerful techniques for teaching adults. Nueva York: JOHN WILEY \& SONS, 2013.

Bruner, Jerome. The Process of Education. Harvard: Harvard University Press, 27 REPRINT, 2003.

CAMARGO, LILIA Y USECHE FIGUEREDO, JUAN DE DIOS. LAS PREGUNTAS COMO HERRAMIENTAS INTELECTUALES PARA EL DESARROLLO DE UN PENSAMIENTO CRÍTICO. REDHECS, V. 20, N. 2, P. 145-156, 2015.

CAPRARO, ROBERT M. Y SLOUGH, SCOTT W. WHY PBL? WHY STEM? WHY NOW? AN INTRODUCTION TO PROJECT-BASED LEARNING: AN INTEGRATED SCIENCE, TECHNOLOGY, ENGINEERING, AND MATHEMATICS (STEM) APPROACH (PP. 1-6). EN ROBERT M. CAPRARO Y SCott W. Slough (Eds.). Project based learning: AN INTEgRated SCIENCE teCHNOlogy ENGINEERING AND MATHEMATICS (STEM) APPROACH. ROTTERDAM: SENSE, 2008. 
CRAFT, ANNA et AL. CREATIVE PRIMARY SCHOOLS: DEVELOPING AND MAINTAINING PEDAGOgy FOR CREATIVITY. ETHNOGRAPHY AND EDUCATION, V. 9, N. 1, P. 16-34, 2014.

DZIB GOODIN, ALMA. LA EVOLUCIÓN DEL APRENDIZAJE: MÁS ALLÁ DE LAS REDES NEURONALES. Revista Chilena de Neuropsicología, v. 8, N. 1, P. 20-25, 2013. DOI: 10.5839/RCNP.2013.0801.04

FAGERBERG, JAN, LANDSTRÖM, HANS Y MARTIN, BEN R. EXPLORING THE EMERGING KNOWLEDGE BASE OF 'THE KNOWLEDGE SOCIETY'. RESEARCH POLICY, V. 41, N. 7, P. 1121-1131, 2012.

FEYERABEND, PAul. SCIENCE In A Free SoCiety. LONDRES: Verso, 1979.

GARRIgós SABATÉ, Júlia y VALERO gARCíA, Miguel. Hablando sobre Aprendizaje Basado en Proyectos con Júlia. REDU. Revista de Docencia UniVERSitARia, v. 10, N. 3, P. 125-151, 2012.

Gellner, ERnest. Spectacles and Predicaments. Cambridge: Cambridge University PRESS, 1979.

GOUVEA, JULIA SVOBODA ET AL. A FRAMEWORK FOR ANALYZING INTERDISCIPLINARY TASKS: IMPLICATIONS FOR STUDENT LEARNING AND CURRICULAR DESIGN. CBE-LIFE SCIENCES EducAtion, v. 12, N. 2, P. 187-205, 2013.

han, Sunyoung, CAPRARO, Robert y CAPRARO, Mary Margaret. How science, TECHNOLOGY, ENGINEERING, AND MATHEMATICS (STEM) PROJECT-BASED LEARNING (PBL) AFFECTS HIGH, MIDDLE, AND LOW ACHIEVERS DIFFERENTLY: THE IMPACT OF STUDENT FACTORS ON ACHIEVEMENT. INTERNATIONAL JOURNAL OF SCIENCE AND MATHEMATICS EDUCATION, V. 13, N. 5, P. 1089-1113, 2015.

hARADA, Violet H., KIRIO, CAROLYN y YAMAMOTO, SANDY. PROJECT-BASED LEARNING: RIGOR AND RELEVANCE IN HIGH SCHOOLS. LIBRARY MEDIA CONNECTION, V. 26, N. 6, P. 14-16, 2008. 
HEYDRICH, MAYRA, ROJAS, MARCIA y HERNÁNDEZ, ANNIA. APRENDIZAJE BASADO EN PROYECTOS: UNA EXPERIENCIA DE INNOVACIÓN DOCENTE. REVISTA UNIVERSIDAD EAFIT, V. 46, N. 158, P. 11-21, 2012.

JENSEN, BJARNE BRUUN Y SIMOVSKA, VENKA. INVOLVING STUDENTS IN LEARNING AND HEALTH PROMOTION PROCESSES: CLARIFYING WHY? WHAT? AND HOW? INTERNATIONAL Journal of Health Promotion and Education, N. 12, P. 150-156, 2005.

Jensen, Bjarne BruUn. A case of two paradigms Within health education. Health EDUCATION RESEARCH, v. 12, N. 4, P. 419-428, 1997.

JOHNSON, CYNTHIA S. y DELAWSKY, SHANNON. PROJECT-BASED LEARNING AND STUDENT engagement. ACADEMic ReSEARCh InTERnAtionAL, V. 4, N. 4, P. 560, 2013.

LEE, JeAn S. ET AL. TAKING A LEAP OF FAITH: REDEFINING TEACHING AND LEARNING IN HIGHER EDUCATION THROUGH PROJECT-BASED LEARNING. INTERDISCIPLINARY JOURNAL OF PROBLEMBASED LEARNING, v. 8, N. 2, P. 2, 2014.

LYALL, CATHERINE Y FLETCHER, ISABEL. EXPERIMENTS IN INTERDISCIPLINARY CAPACITYBUILDING: THE SUCCESSES AND CHALLENGES OF LARGE-SCALE INTERDISCIPLINARY INVESTMENTS. SCIENCE AND Public Policy, v. 40, N. 1, P. 1-7, 2013.

MIHARDI, SATRIA, HARAHAP, MARA BANGUn y SANI, RIDWAN ABDULLAH. THE EFFECT OF Project Based Learning Model With KWL WorkSheet on StUdent CREATIVE Thinking Process in Physics Problems. Journal of Education and Practice, v. 4, N. 25, P. 188200,2013

MORAleS, Claudia y tORRES, Amauri. AprendizAJe basado en proyectos para el desarrollo de competencias. Revista Iberoamericana de Producción Académica y Gestión Educativa, v. 2, N. 35, P. 1-10, 2015.

MUJikA, Mikel Garmendia et al. Proyecto de formación del profesorado universitario de Ciencias, Matemáticas y Tecnología, en las metodologías de Aprendizaje Basado en Problemas y PROyectos. EnSEÑANZA DE LAS CIENCIAS: REVISTA DE INVESTIGACIÓN Y EXPERIENCIAS DIDÁCTICAS, V. 32, N. 2, P. 113-129, 2014. 
NORDIN, LONE LINDEGAARD. IMPLEMENTING THE HEALTH PROMOTING SCHOOL IN DENMARK: A CASE STUdY. HEALTH EdUCATION, v. 116, N. 1, P. 86-103, 2016.

NOTARI, Michele, BAUMGARTNER, AdRIAN y HERZOG, WALTER. SOCIAL SKILLS AS PREDICTORS OF COMMUNICATION, PERFORMANCE AND QUALITY OF COLLABORATION IN PROJECTBASED LEARNING. JOURNAL OF COMPUTER ASSISTED LEARNING, v. 30, N. 2, P. 132-147, 2014.

OChOA, Rafael Flórez. Estrategias de enseñanza y pedagogía. Actualidades PEdAGógICAS, N. 61, P. 15-26, 2013.

PENNINGTON, DEANA D. ET AL. TRANSDISCIPLINARY RESEARCH, TRANSFORMATIVE LEARNING, AND TRANSFORMATIVE SCIENCE. BIOSCIENCE, V. 63, N. 7, P. 564-573, 2013.

PREETI, BAJAJ, ASHISH, AHUJA y SHRIRAM, GosaVI. PROBLEM BASEd lEARNING (PBL)-AN EFFECTIVE APPROACH TO IMPROVE LEARNING OUTCOMES IN MEDICAL TEACHING. JOURNAL OF CLINICAL AND DIAGNOSTIC RESEARCH: JCDR, V. 7, N. 12, P. 2896, 2013.

RAVITZ, JASON ET AL. USING PROJECT BASED LEARNING TO TEACH 21ST CENTURY SKILLS: Findings from a stateWide initiative. En AnNual Meetings of the American Educational Research Association, April. Vancouver, BC, 2012. ReCuperado de: HTTP://WWW.BIE. ORG/RESEARCH/STUDY/PBL_21CS_WV. ACCESO EN: 15 JUNIO 2016.

SAAVEDRA, ANNA ROSEFSKY Y OPFER, V. DARLEEN. LEARNING 21ST-CENTURY SKILLS REQUIRES 21St-CENTURY TEACHING. Phi DeltA KAPPAN, v. 94, N. 2, P. 8-13, 2012.

SilVA, ORMEnzina G. y NAVARRO, Elaine Cristina. A RELAÇÃo PROFESSOR-ALUNO NO PROCESSO ENSINO-APRENDIZAGEM. REVISTA ELETRONICA DA UNIVAR, V. 3, N. 8, P. 95-100, 2012.

SINEK, SIMON. LA CLAVE ES EL PORQUÉ. CÓMO LOS GRANDES LÍDERES NOS INSPIRAN A ACTUAR. Barcelona: PenÍNSUla, 2009.

STEFANOU, CANDice ET AL. SELF-REgULATION AND AUtONOMY IN PROBLEM-AND PROJECTPágina | 59 BASED LEARNING ENVIRONMENTS. ACTIVE LEARNING IN HIGHER EDUCATION, V. 14, N. 2, P. 109$122,2013$. 
PROJeCtS. Education In the KNOWLEdge SOCIETy, V. 17, N. 1, P. 19, 2016.

Recebido: 2016-07-15

Aprovado: 2017-09-26

DOI: $10.3895 /$ rbect.v10n3.4454

Como citar:

BAUTISTA-VALLEJO, J. M.; ESPIGARES-PINAZO, M. J.; HERNÁNDEZ-CARRERA, R. M. Aprendizaje Basado en Proyectos (ABP) ante el reto de una nueva enseñanza de las ciencias. Revista Brasileira de Ensino de Ciência e Tecnologia, v. 10, n. 3, 2017. Disponível em: <https://periodicos.utfpr.edu.br/rbect/article/view/4454>. Acesso em: xxx.

Correspondência:

José Manuel Bautista-Vallejo - bautista@uhu.es.

Direito autoral: Este artigo está licenciado sob os termos da Licença Creative Commons-Atribuição 4.0 Internacional.

\section{(c) (1)}

